\title{
Fuzzy Sets for Modelling Fineness Perception in Texture Images
}

\author{
J. Chamorro-Martínez, E. Galán-Perales, and J.M. Soto-Hidalgo \\ Department of Computer Science and Artificial Intelligence, University of Granada \\ C/ Periodista Daniel Saucedo Aranda s/n, 18071 Granada, Spain \\ e-mail : \{jesus,elena,soto\}@decsai.ugr.es
}

\begin{abstract}
Fineness is a primary texture feature frequently used for image content description. However, it is an ambiguous concept difficult to be characterized. To face this ambiguity, we propose to model the fineness by means of fuzzy sets, relating a representative fineness measure (our reference set) with the human perception of fineness. In our study, a wide variety of measures have been analyzed, defining a fuzzy set for each measure. The fineness perception has been collected from polls filled by human subjects, performing an aggregation of their assessments by means of OWA operators. For a given measure, the corresponding membership function is obtained by fitting the collected data. The performance of each fuzzy set is analyzed and checked with the human assessments, proposing a subgroup of them as the most adequate for modelling fineness perception in texture images.
\end{abstract}

Keywords: Image features, textural features, fuzzy texture, human perception, coarseness, fineness.

\section{INTRODUCTION}

Texture is one of the main features used in visual-based systems to describe the content of an image [1], [2]. As well as being an important feature, it is also one of the most difficult to be characterized due to the imprecision of the concept itself [3], [4]. In fact, there is not an accurate definition for the concept of texture but some widespread intuitive ideas. In this way, texture is described by some authors as local changes in the intensity patterns or gray tones which is used in opposition to the homogeneity idea. Other authors consider texture as a set of basic items called texels (or texture primitives), arranged in a certain way [5].

Furthermore, it is usual for humans to describe visual textures according to some vague "textural concepts" like coarseness/fineness, orientation or regularity [6], [7], [8]. From all of them, the coarseness/fineness is the most popular one, being common to associate the presence of fineness with the presence of texture (from this point of view, texture is defined as local variations against the idea of homogeneity). In this sense, a fine texture is considered as small texture primitives with big gray tone differences between neighbor primitives (e.g. the image in figure 1(A)), whereas a coarse texture corresponds to bigger primitives formed by several pixels (e.g. the image in figure 1(I)). By considering the importance of this textural concept, in this paper we will focus our study on the "fineness" modelling (let us remark that

\footnotetext{
${ }^{1}$ This work has been supported by the MEC under the TEC2006-13845 project
}

"coarseness" and "fineness" are opposite but related textural concepts).

The own imprecision of the concept of texture suggests the use of representation models that incorporate the uncertainty. Nevertheless, the majority of the approaches that can be found in the literature are crisp proposals [9], [10], [5], [6] where uncertainty is not properly taken into account. To face this problem, fuzzy logic has been recently employed for representing the imprecision related to texture. However, in many of these approaches, fuzzy logic is usually applied just during the process but the output do not habitually model the imprecision (being often a crisp one). Examples of this fact are frequently found in the literature, like those approaches that use texture to perform image segmentation or classification on the basis of fuzzy clustering [11], [12], [13], fuzzy rules [14], [15], [16], etc.

Other interesting approaches emerge from the content-based image retrieval scope, where semantic data is managed by means of fuzzy sets [17], [18]. In these proposals, a mapping from low-level statistical features to high level textural concepts is performed by defining membership functions for each textural feature. However, given a feature, these membership functions are not obtained by considering the relationship between the computational feature and the human perception of texture, so the linguistic labels related to these membership functions do not necessarily match what a human would expect.

In this paper we propose to solve this problem by representing the concept of fineness on the basis of fuzzy sets relating representative measures of the modelled texture (usually some statistic) with its human perception of fineness. In our analysis, a wide variety of measures have been considered, including classical statistical measures, frequency domain and fractal dimension measures, etc. In addition, linear, quadratic and cubic spline functions have been considered in order to define the membership function. Thus, this paper propose a group of fuzzy sets (defined on different measures) which model the textural fineness according to the human perception. The performance of each fuzzy set is analyzed and checked with the human assessments, proposing a subgroup of them as the most adequate for modelling fineness perception in texture images.

The rest of the paper is organized as follows. In section II we introduce our methodology to obtain the fuzzy sets that 

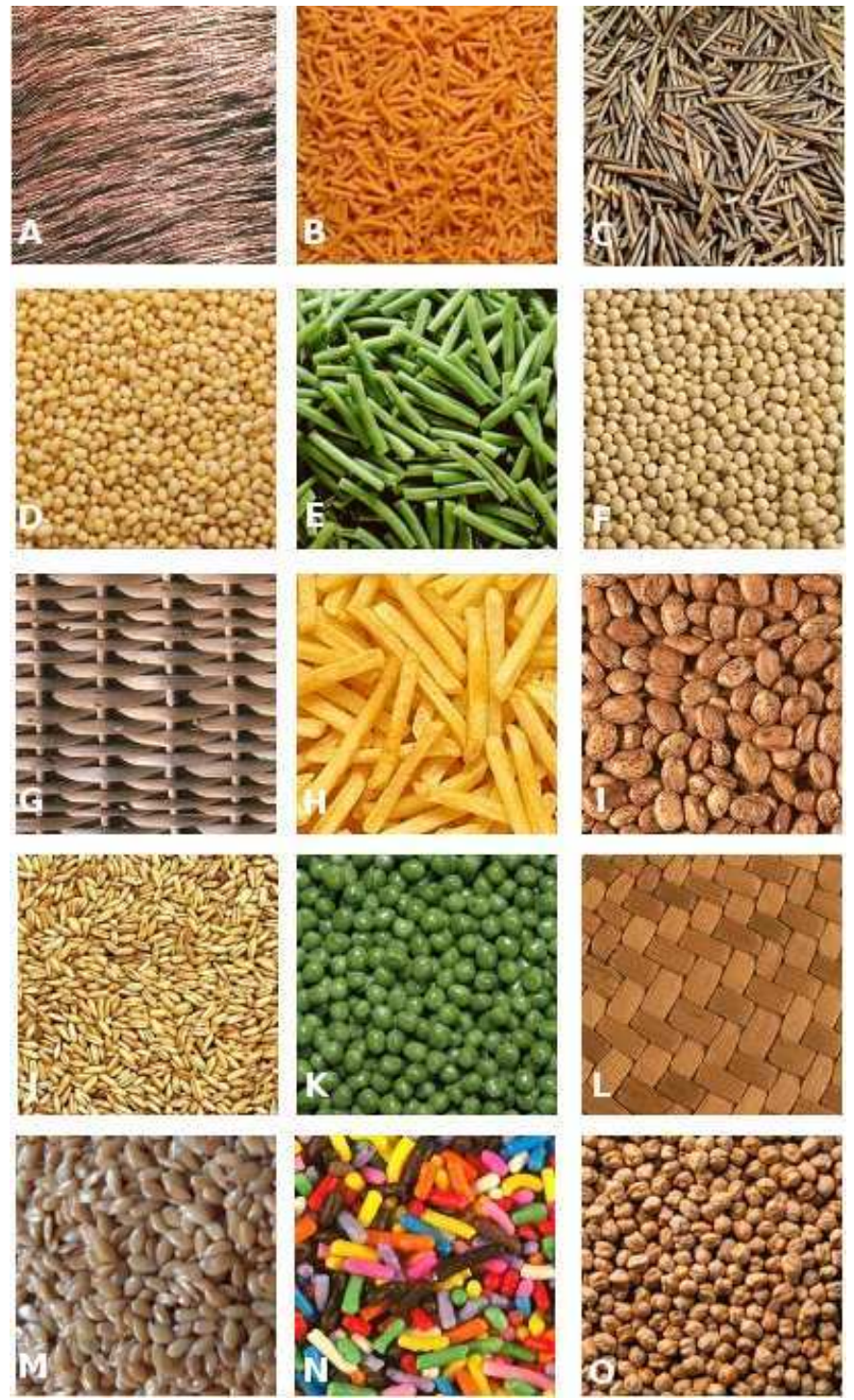

Fig. 1. Some examples of images with different degrees of fineness

model the fineness textural concept. In section III we show the results of applying the models and the main conclusions and future work are summarized in section IV.

\section{FuZZY MODELling OF FINENESS}

There are many measures in the literature that, given an image, capture the fineness (or coarseness) presence in the sense that the greater the value given by the measure, the greater the perception of texture [5]. However, there is no perceptual relationship between the value given by these measures and the degree in which the humans perceive the texture. Thus, given a certain value calculated by applying a measure to an image, there is not an immediate way to decide whether there is a fine texture, a coarse texture or something intermediate (i.e. there is not a textural interpretation).

To face this problem, we propose to model the fineness perception as a fuzzy set defined on the domain of a given measure. For this purpose, two questions will be faced: (i) what reference set should be used for the fuzzy set, and (ii) how to obtain the related membership functions.

As reference set, a wide variety of measures of fineness will be considered. Concretely, we have selected the 18 measures indicated in the first column of table I, that includes classical statistical measures well known in the literature, measures in the frequency domain, fractal dimension analysis, etc. All of them are automatically computed from the texture image. From now on, we will note $\mathcal{P}=\left\{P_{1}, \ldots, P_{K}\right\}$ the set of measures of fineness and $\mathcal{T}_{k}$ the fuzzy set defined on the domain of a given $P_{k} \in \mathcal{P}$.

To obtain the membership function ${ }^{1}$ of the fuzzy set $\mathcal{T}_{k}$, a functional relationship between the measure $P_{k}$ and the presence degree of fineness related to it will be learnt. To do it, we will use a set $\mathcal{I}=\left\{I_{1}, \ldots, I_{N}\right\}$ of $N$ images that fully represent the different degrees of fineness. Thus, for each image $I_{i} \in \mathcal{I}$, we will obtain (a) a value calculated applying the measure $P_{k} \in \mathcal{P}$ to the image $I_{i}$, noted as $m_{k}^{i}$, and (b) a human assessment of the fineness degree perceived, noted as $v^{i}$, which will be collected by means of a poll with human subjects (section II-A). From the multiset $\Psi_{k}=\left\{\left(m_{k}^{1}, v^{1}\right), \ldots,\left(m_{k}^{N}, v^{N}\right)\right\}$, the membership function $\mathcal{T}_{k}$ will be estimated (section II-B).

\section{A. Assessment collection}

In this section, the way to obtain a vector $\Gamma=\left[v 1, \ldots, v^{N}\right]$ of the assessments of the perception degree of fineness from the image set $\mathcal{I}=\left\{I_{1}, \ldots, I_{N}\right\}$ will be described. Thus, firstly the image set $\mathcal{I}$ will be selected. After that, a poll which allows to get assessments of the perception degree of fineness will be designed. These assessments will be obtained for each image in $\mathcal{I}$, so an aggregation of the different assessments will be performed.

1) The texture image set: A set $\mathcal{I}=\left\{I_{1}, \ldots, I_{N}\right\}$ of $N=$ 80 images representative of the concept of fineness has been selected. Figure 1 shows some images extracted from the set $\mathcal{I}$. The selection was done to cover the different perception degrees of fineness with a representative number of images. Furthermore, the images have been chosen so that as far as possible, just one perception degree of fineness is perceived.

2) The poll: Given the image set $\mathcal{I}$, the next step is to obtain assessments about the perception of fineness from a set of subjects. From now on we shall note as $\Theta^{i}=\left[o_{1}^{i}, \ldots, o_{L}^{i}\right]$ the vector of assessments obtained from $L$ subjects for the image $I_{i}$. To get $\Theta^{i}$, subjects will be asked to assign images to classes, so that each class has associated a perception degree of fineness.

In particular, 20 subjects have participated in the poll and 9 classes have been considered. The first nine images in figure 1 show the nine representative images for each class used in this poll. It should be noticed that the images are decreasingly ordered according to the presence degree of the fineness concept. The first class (Figure 1(A)) represents a presence

\footnotetext{
${ }^{1}$ To simplify the notation, as it is usual in the scope of fuzzy sets, we will use the same notation $\mathcal{T}_{k}$ for the fuzzy set and for the membership function that defines it
} 
TABLE I

FITTING RMSE AND PARAMETER VALUES THAT DEFINE THE MEMBERSHIP FUNCTION RELATED TO EACH MEASURE

\begin{tabular}{|l|c|c|c|c|c|c|c|}
\hline \multicolumn{2}{|c|}{} & \multicolumn{3}{|c|}{ Parameter values } & \multicolumn{2}{c|}{ Knots } \\
\hline Measures & RMSE & $a_{3}$ & $a_{2}$ & $a_{1}$ & $a_{0}$ & $\alpha$ & $\beta$ \\
\hline Tamura [6] & 0.2240 & - & -0.247 & 0.822 & 0.421 & 3.77 & 2.31 \\
Fractal dimension [19] & 0.2243 & - & - & 1.05 & -2.36 & 2.25 & 3.2 \\
Edge density [20] & 0.2483 & 265.4 & -207.8 & 57.17 & -5.08 & 0.177 & 0.364 \\
Amadasun [7] & 0.2546 & -0.0007 & 0.0282 & -0.376 & 2.22 & 20.3 & 4.66 \\
Correlation [5] & 0.2555 & - & - & -1.15 & 1.12 & 0.978 & 0.106 \\
Local Homogeneity [5] & 0.3022 & -953 & 395 & -54.7 & 2.9 & 0.211 & 0.0514 \\
SNE [21] & 0.3065 & 51.5 & -110 & 77.8 & -18 & 0.511 & 0.935 \\
Weszka [22] & 0.3159 & 106 & -42.7 & 9.87 & -0.132 & 0.0142 & 0.203 \\
Newsam [23] & 0.3179 & 0.0306 & -1.71 & 31.9 & -198 & 16.2 & 21.3 \\
Wu [24] & 0.3186 & $6 \cdot 10^{-6}$ & -0.0006 & 0.0422 & -0.119 & 2.95 & 42.7 \\
Short Run Emphasis [25] & 0.3349 & 17900 & -52000 & 50300 & -16200 & 0.94 & 1 \\
Contrast [5] & 0.3459 & $9 \cdot 10^{-13}$ & $-3 \cdot 10^{-8}$ & 0.0003 & -0.024 & 70.6 & 5420 \\
Entropy [5] & 0.3622 & 1.22 & -31.6 & 273 & -785 & 8.02 & 9.44 \\
Uniformity[5] & 0.3802 & $-5 \cdot 10^{10}$ & $5 \cdot 10^{7}$ & -17900 & 2.19 & 0.0005 & $9 \cdot 10^{-5}$ \\
Variance[5] & 0.3987 & - & - & $5 \cdot 10^{-5}$ & -0.267 & 5880 & 27800 \\
DGD [26] & 0.4073 & 0.0001 & -0.0104 & 0.205 & -0.194 & 1 & 14.1 \\
FMPS [27] & 0.4127 & 0.005 & -0.081 & -1.62 & 27.2 & 18.2 & 19.9 \\
Abbadeni [28] & 0.4928 & - & - & 98.5 & -49.4 & 0.502 & 0.512 \\
\hline
\end{tabular}

degree of 1 while the ninth class (Figure 1(I)), represents a presence degree of 0 . The rest of the classes (Figure 1(B)-(H)) represent degrees in the interval $(0,1)$.

As result, a vector of 20 assessments $\Theta^{i}=\left[o_{1}^{i}, \ldots, o_{20}^{i}\right]$ is obtained for each image $I_{i} \in \mathcal{I}$. The degree $o_{j}^{i}$ associated to the assessment given by the subject $S_{j}$ to the image $I_{i}$ is computed as $o_{j}^{i}=(9-k) * 0.125$, where $k \in\{1, \ldots, 9\}$ is the index of the class $C_{k}$ to which the image is assigned by the subject.

3) Assessment aggregation: Our aim at this point is to obtain, for each image in the set $\mathcal{I}$, one assessment $v^{i}$ that summarizes the assessments $\Theta^{i}$ given by the different subjects about the presence degree of fineness.

To aggregate opinions we have used an OWA operator guided by a quantifier [29]. Concretely, the quantifier "the most" has been employed, which allows to represent the opinion of the majority of the polled subjects. This quantifier is defined as

$$
Q(r)=\left\{\begin{array}{lr}
0 & \text { if } r<a \\
\frac{r-a}{b-a} & \text { if } a \leq r \leq b \\
1 & \text { if } r>b
\end{array}\right.
$$

with $r \in[0,1], a=0.3$ and $b=0.8$. Once the quantifier $Q$ has been chosen, the weighting vector of the OWA operator can be obtained following Yager [29] as $w_{j}=Q(j / L)-Q((j-$ $1) / L), j=1,2, \ldots, L$. According to this, for each image $I_{i} \in$ $\mathcal{I}$, the vector $\Theta^{i}$ obtained from $L$ subjects will be aggregated into one assessment $v^{i}$ as follows:

$$
v^{i}=w_{1} \hat{o}_{1}^{i}+w_{2} \hat{o}_{2}^{i}+\ldots+w_{L} \hat{o}_{L}^{i}
$$

where $\left[\hat{o}_{1}^{i}, \ldots, \hat{o}_{L}^{i}\right]$ is a vector obtained by ranking in nonincreasing order the values of the vector $\Theta^{i}$.

\section{B. Fitting the membership function}

At this point, the aim is to obtain, for each measure $P_{k} \in$ $\mathcal{P}$, the corresponding membership function $\mathcal{T}_{k}$. Since we are searching for a function which associates the measures values $\left(m_{k}^{i}\right)$ and the human assessments of fineness $\left(v^{i}\right)$, we propose to estimate $\mathcal{T}_{k}$ by means of a Least Squares Fitting of the multiset $\Psi_{k}=\left\{\left(m_{k}^{1}, v^{1}\right), \ldots,\left(m_{k}^{N}, v^{N}\right)\right\}$.

To define $\mathcal{T}_{k}$, the following considerations will be taked into account:

- $\mathcal{T}_{k}$ should be a monotonic function

- The values $\mathcal{T}_{k}(x)=0$ and $\mathcal{T}_{k}(x)=1$ should be achieved from a certain value

Regarding the above properties, in this paper we propose to define $\mathcal{T}_{k}$ as a function

$$
\mathcal{T}_{k}: \mathbb{R} \rightarrow[0,1]
$$

of the form ${ }^{2}$ (see figure 2)

$\mathcal{T}_{k}\left(x ; a_{n} \ldots a_{0}, \alpha, \beta\right)= \begin{cases}0 & x<\alpha \\ \operatorname{poly}^{n}\left(x ; a_{n} \ldots a_{0}\right) & \alpha \leq x \leq \beta, \\ 1 & x>\beta\end{cases}$

with $\operatorname{poly}^{n}\left(x ; a_{n} \ldots a_{0}\right)$ being a polynomial function

$$
\operatorname{poly}^{n}\left(x ; a_{n} \ldots a_{0}\right)=a_{n} x^{n}+\ldots+a_{1} x^{1}+a_{0}
$$

\footnotetext{
${ }^{2}$ Note that this function is defined for measures that increase according to the perception of fineness but for those that decreases, the function needs to be changed appropriately
} 


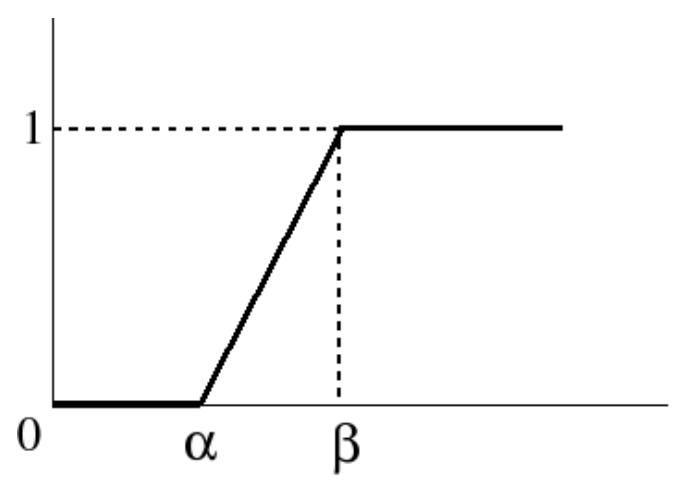

Fig. 2. Graphical representation of the kind of membership functions used in our approach (only the linear case is plotted)

In our proposal, the parameters $a_{n} \ldots a_{0}, \alpha$ and $\beta$ of the function $\mathcal{T}_{k}$ are calculated by carrying out a Least Squares Fitting on $\Psi_{k}$ taking into account the restriction of obtaining a monotonic function and considering $\mathrm{n}=1,2,3$ (i.e. linear, quadratic and cubic functions) to define the polynomial function. Table I shows for each measure $P_{k} \in \mathcal{P}$ the least RMSE fitting error obtained, the corresponding parameters $\left(a_{3}, \ldots, a_{0}\right)$ and the knot values $\alpha$ and $\beta$ necessary to define the spline function. Note that the fitting error can be viewed as a goodness measure of the ability of the measure to represent the perception of fineness.

Table I has been sorted in increasing order of the errors and it can be noticed that the five first measures obtain a similar RMSE which difference is as much of 0.03 what implies that these measures will give similar results when employed. Furthermore, the last measures appearing in table I have associated a high RMSE very distant from the above mentioned measures which implies that these measures are not providing a representative information about the perception of fineness.

\section{RESULTS}

In this section, the membership function $\mathcal{T}_{k}$ obtained for each measure $P_{k}$ (and defined by the parameter values shown in table I) will be applied in order to analyze the performance of the proposed model. For this purpose, some experiments using images with different associated fineness presence degrees will be performed, analyzing the goodness of the fuzzy sets for each case.

Table II shows three real images with different perception degree of fineness. For each image and each measure, this table shows the value obtained by the related function and the error obtained when comparing this value with the assessment value given by subjects (by computing the difference between both of them). It can be noticed by looking the results shown in this table that our model allows to represent appropriately the perception of fineness. Note that the values given by the membership functions are similar to the corresponding assessment degree for most of the obtained functions.

The set of measures are presented in Table II following the same order as in table I. As it was mentioned above, smaller
TABLE II

THREE REAL IMAGES WITH DIFFERENT PERCEPTION DEGREE OF FINENESS FOR EACH MEASURE, THE MEMBERSHIP DEGREE IS SHOWED TOGETHER WITH ITS ERROR

\begin{tabular}{|c|c|c|c|c|c|c|}
\hline \multirow[b]{2}{*}{ Measure } & \multicolumn{2}{|c|}{$\begin{array}{c}\text { Assessment } \\
\text { Value }=0\end{array}$} & \multicolumn{2}{|c|}{$\begin{array}{c}\text { Assessment } \\
\text { Value }=0.5\end{array}$} & \multicolumn{2}{|c|}{$\begin{array}{c}\text { Assessment } \\
\text { Value }=1\end{array}$} \\
\hline & Value & Error & Value & Error & Value & Error \\
\hline Tamura & 0 & 0 & 0.545 & 0.045 & 0.966 & 0.034 \\
\hline FD & 0 & 0 & 0.594 & 0.094 & 1 & 0 \\
\hline ED & 0 & 0 & 0.488 & 0.012 & 1 & 0 \\
\hline Amadasun & 0 & 0 & 0.489 & 0.011 & 0.854 & 0.146 \\
\hline Corr. & 0.012 & 0.012 & 0.475 & 0.025 & 1 & 0 \\
\hline LH & 0 & 0 & 0.459 & 0.041 & 0.735 & 0.265 \\
\hline SNE & 0 & 0 & 0.421 & 0.079 & 0.843 & 0.157 \\
\hline Weszka & 0.085 & 0.085 & 0.572 & 0.072 & 0.741 & 0.259 \\
\hline Newsam & 0 & 0 & 0.435 & 0.065 & 0.605 & 0.395 \\
\hline $\mathrm{Wu}$ & 0.076 & 0.076 & 0.607 & 0.107 & 0.774 & 0.226 \\
\hline SRE & 0 & 0 & 0.548 & 0.048 & 0.709 & 0.291 \\
\hline Contrast & 0.028 & 0.028 & 0.634 & 0.134 & 0.604 & 0.396 \\
\hline Entropy & 0.285 & 0.285 & 0.687 & 0.187 & 0.484 & 0.516 \\
\hline Uniformity & 0.115 & 0.115 & 0.820 & 0.320 & 0.663 & 0.337 \\
\hline Variance & 0.271 & 0.271 & 0.640 & 0.140 & 0.743 & 0.257 \\
\hline DGD & 0 & 0 & 0.331 & 0.169 & 1 & 0 \\
\hline FMPS & 0.360 & 0.360 & 0.717 & 0.217 & 0.803 & 0.197 \\
\hline Abbadeni & 0.396 & 0.396 & 0.410 & 0.09 & 0.596 & 0.404 \\
\hline
\end{tabular}

error values are found for the first set of five measures while higher error values are found if we look at the last rows in table II. It can also be noticed that measures do not necessarily keep strictly the same order as shown in table I but a general increase of the error is found when the table II is looked from top to bottom rows.

Let's consider figure 3(A) corresponding to a mosaic made by several images, each one with a different increasing perception degree of fineness. The perception degree of fineness for each subimage has been calculated using the Tamura measure (the one with least fitting error according to table I) and the results are shown in figure 3(B) where a white grey level means maximum perception of fineness, while a black one corresponds to no perception of fineness (the numeric value is also shown on each subimage). It can be noticed that our model captures the evolution of the perception degrees of fineness.

Table III shows a comparative between our model and the assessments obtained from subjects for the images in figure 3 . To get such asessments, showed in the second column in table III, we have aggregated the assessments of 20 subjects following the steps explained in section II-A. The third column shows the fineness degree obtained by applying our model, the fourth column shows the difference between the computed degree and the human assessment. In the case of the fifth column we calculate the differences between the assessment given by each subject and the computed degree, and we obtain as error measure the mean from these 20 differences. Finally, the average errors shown in the last row with values of 0.011 and 0.029 show the goodness of our approach to represent the 

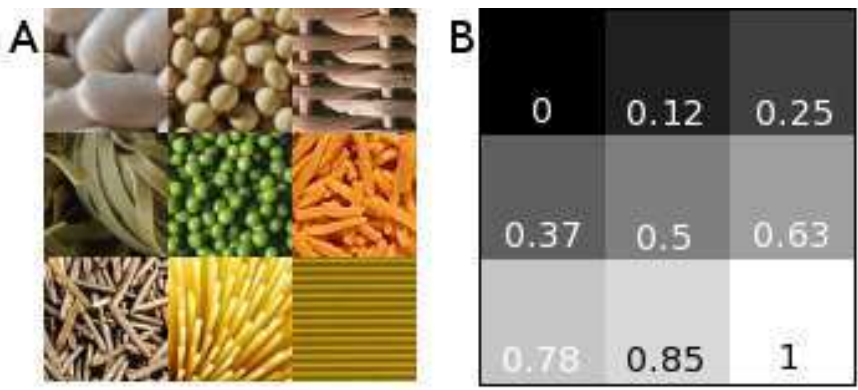

Fig. 3. Results for a mosaic image. (A) Original mosaic image (B) Degree of fineness obtained with the proposed model

TABLE III

ERRORS OBTAINED FROM THE MOSAIC IMAGE OF FIGURE 3

\begin{tabular}{|c|c|c|c|c|}
\hline Image & $\begin{array}{c}\text { Human } \\
\text { Assessment(H) }\end{array}$ & $\begin{array}{l}\text { Estimated } \\
\text { Value }(\mathrm{V})\end{array}$ & $\begin{array}{c}\text { Error \#1 } \\
(|H-V|)\end{array}$ & Error \#2 \\
\hline 1 & 0 & 0 & 0 & 0 \\
\hline 2 & 0.125 & 0.125 & 0 & 0.021 \\
\hline 3 & 0.250 & 0.250 & 0 & 0.010 \\
\hline 4 & 0.375 & 0.375 & 0 & 0.094 \\
\hline 5 & 0.500 & 0.500 & 0 & 0.010 \\
\hline 6 & 0.625 & 0.625 & 0 & 0.052 \\
\hline 7 & 0.750 & 0.780 & 0.030 & 0.010 \\
\hline 8 & 0.875 & 0.852 & 0.023 & 0.010 \\
\hline 9 & 1.000 & 0.960 & 0.040 & 0.052 \\
\hline & & & Avg: 0.011 & Avg: 0.029 \\
\hline
\end{tabular}

subjectivity found in fineness perception.

Figure 4 presents an example where the proposed fuzzy sets have been employed for pattern recognition. Concretely, the figure shows a microscopy image (Figure 4(A)) corresponding to the microstructure of a metal sample [30]. The lamellae indicates islands of eutectic, which are to be separated from the uniform light regions. The brightness values in regions of the original image are not distinct, so texture information is needed for extracting the uniform areas. This fact is showed in Figure $4(\mathrm{~B} 1, \mathrm{~B} 2)$, where a thersholding on the original image is displayed (homogeneous regions cannot be separated from the textured ones as they "share" brightness values).

Figure 4(C1) shows a mapping from the original image to its "fineness" membership degree (in this example, the fuzzy set corresponding to the edge density measure has been used). For each pixel in the original image, a centered window of size $15 \times 15$ has been analyzed and its fineness membership degree has been calculated. Thus, Figure 4(C1) represents the degree in which the human perceives the texture, with a white grey level meaning maximum perception of fineness, and a black one meaning no perception of fineness (i.e., maximum perception of coarseness). It can be noticed from Figure $4(\mathrm{C} 1)$ that uniform regions correspond to areas with low degrees of fineness (i.e., high coarseness), so if only the pixels with fineness degree lower than 0.1 are selected (which it is equivalent to a coarseness degree upper than 0.9), the uniform light regions emerge with ease (Figure 4(C2,C3)).
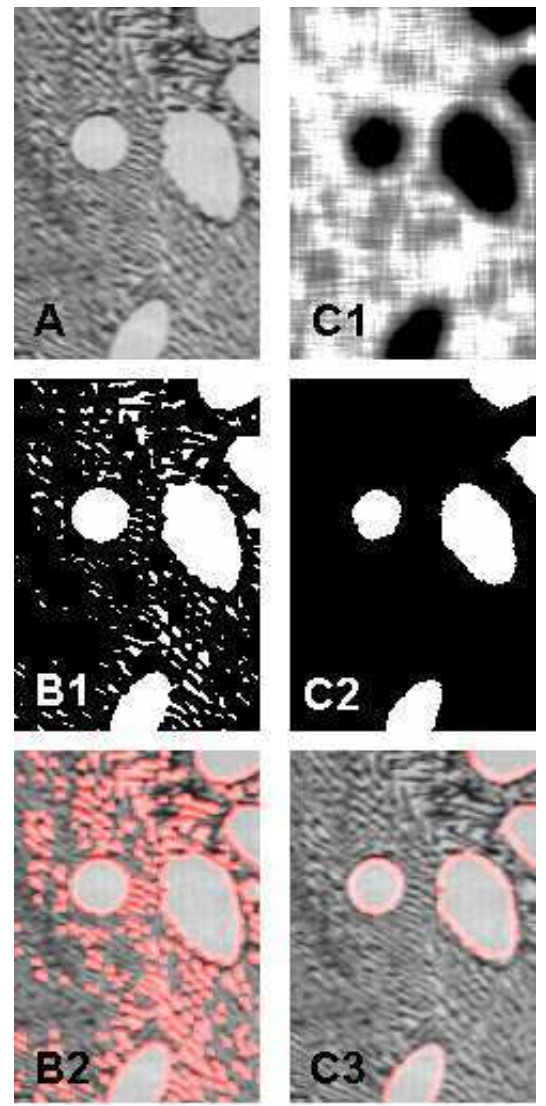

Fig. 4. Example of pattern recognition using the proposed model (A) Original image (B1) Binary image obtained by thresholding the original image (B2) Region outlines of B1 superimposed on original image (C1) Fineness membership degrees obtained with our model from the original image (C2) Binary image obtained by inverting the $\alpha$-cut on $\mathrm{C} 1$ with $\alpha=0.1$ (C3) Region outlines of $\mathrm{C} 2$ superimposed on original image

\section{CONClusions AND FUtURE WORKS}

In this paper, fuzzy sets for fineness perception have been defined on the domain of certain measures. The membership function associated to the fuzzy set has been learnt by finding the functional relationship between a certain measure (automatically computed over the image) and the presence degree of the textural concept. The measures have been chosen so that they collect information of the textural fineness under study whereas the presence degree has been obtained by performing a poll with humans and aggregating their assessments by means of OWA operators.

Satisfactory performance has been found when using the different fuzzy sets with real images and the results show a high level of connection with the assessments given by subjects. From the different measures used in this paper, we conclude there is a set of them (the five first measures shown in table I) that give similar results when their associated fuzzy set is employed. Moreover, from this table it is also concluded there are other measures (the ones found in the last rows) that obtain worse results, being less advisable their employment.

As future work, we will extend the proposed approach to obtain fuzzy sets on $\mathbb{R}^{n}$, i.e. membership functions defined 
on vectors of measures. Furthermore, the performance of the fineness functions will be analyzed in applications like textural classification or segmentation.

\section{REFERENCES}

[1] M. Flicker, H. Sawhney, W. Niblack, J. Ashley, Q. Huang, B. Dom, M. Gorkani, J. Hafner, D. Lee, D. Petkovic, D. Steele, and P. Yanker, "Query by image and video content: the qbic system," IEEE Computer, vol. 28, no. 9, pp. 23-32, 1995.

[2] Q. Iqbal and J. K. Aggarwal, "Cires: A system for content-based retrieval in digital image libraries," in Proc. of International Conference on Control, Automation, Robotics and Vision, vol. 2, Singapore, December 2002, pp. 205-210.

[3] C. Jacobs, A. Finkelstein, and D. Salesin, "Fast multiresolution image querying," in Proc. Computer Graphics, Annual Conference Series, 1995, pp. 277-286.

[4] S. Belongie, C. Carson, H. Greenspan, and J. Malik, "Color- and texturebased image segmentation using expectation-maximization and its application to content-based image retrieval," in Proc. 6th International Conference on Computer Vision, 1998, pp. 675-682.

[5] R. Haralick, "Statistical and structural approaches to texture," Proceedings IEEE, vol. 67, no. 5, pp. 786-804, 1979.

[6] H. Tamura, S. Mori, and T. Yamawaki, "Textural features corresponding to visual perception," IEEE Trans. on Systems, Man and Cybernetics, vol. 8, pp. 460-473, 1978 .

[7] M. Amadasun and R. King, "Textural features corresponding to textural properties," IEEE Transactions on Systems, Man and Cybernetics, vol. 19 , no. 5, pp. 1264-1274, 1989.

[8] A. Rao and G. Lohse, "Identifying high level features of texture perception," Graphical Models and Image Processing, vol. 55, no. 3, pp. 218-233, 1993.

[9] T. R. Reed and J. H. D. Buf, "A review of recent texture segmentation and feature extraction techniques," CVGIP: Image Understanding, vol. 57, no. 3, pp. 359-372, 1993.

[10] K. Chang, K. Bowyer, and M. Sivagurunath, "Evaluation of texture segmentation algorithms," in Proc. IEEE Computer Society Conference on Computer Vision and Pattern Recognition, vol. 57, 2003, pp. 294299.

[11] C. Wang, H. Wang, and Q. Mei, "Texture segmentation based on an adaptively fuzzy clustering neural network," in Proc. of 2004 International Conference on Machine Learning and Cybernetics, vol. 2, 2004, pp. 1173-1176.

[12] M. Hanmandlu, V. K. Madasu, and S. Vasikarla, "A fuzzy approach to texture segmentation," in Proc. International Conference on Information Technology: Coding and Computing, vol. 1, 2004, pp. 636-642.

[13] A. Shackelford, "A hierachical fuzzy classification approach for highresolution multispectral data over urban areas," IEEE Transactions on Geoscience and Remote Sensing, vol. 41, no. 9, pp. 1920-1932, 2003.

[14] M. Potrebić, "Iterative fuzzy rule base technique for image segmentation," in Proc. of 7th seminar on Neural Network Applications in Elecrical Engineering, 23-25 Sept. 2004, pp. 221-224.

[15] X. Dai and J. Maeda, "Unsupervised segmentation of textured color images using fuzzy homogeneity decision," in Proc. 4th International Symposium on Video/Image Processing and Multimedia Communications, 2002, pp. 75-78.

[16] G. Karmakar, L. Dooley, and M. Murshed, "Fuzzy rule for image segmentation incorporating texture features," in Proc. of 2002 International Conference on Image Processing, vol. 1, 22-25 Sept. 2002, pp. I-797I-800.

[17] S. Kulkarni and B. Verma, "Fuzzy logic based texture queries for cbir," in Proc. 5th International Conference on Computational Intelligence and Multimedia Applications, 2003, pp. 223-228.

[18] H. Lin, C. Chiu, and S. Yang, "Finding textures by textual descriptions, visual examples, and relevance feedbacks," Pattern Recognition Letters, vol. 24, no. 14, pp. 2255-2267, 2003.

[19] S. Peleg, J. Naor, R. Hartley, and D. Avnir, "Multiple resolution texture analysis and classification," IEEE Transactions on Pattern Analysis and Machine Intelligence, no. 4, pp. 518-523, 1984.

[20] J. Canny, "A computational approach to edge detection," IEEE Transactions on Pattern Analysis and Machine Intelligence, vol. 8, no. 6, pp. 679-698, 1986.
[21] C. Sun and W. Wee, "Neighboring gray level dependence matrix for texture classification," Computer Vision, Graphics and Image Processing, vol. 23, pp. 341-352, 1983.

[22] J. Weszka, C. Dyer, and A. Rosenfeld, "A comparative study of texture measures for terrain classification," IEEE Trans. on SMC, vol. 6, pp. 269-285, 1976.

[23] S. Newsam and C. Kammath, "Retrieval using texture features in high resolution multi-spectral satellite imagery," in Data Mining and Knowledge Discovery: Theory, Tools, and Technology VI, SPIE Defense and Security, April 2004.

[24] C. Wu and Y. Chen, "Statistical feature matrix for texture analysis," CVGIP: Graphical Models and Image Processing, vol. 54, no. 5, pp. 407-419, 1992.

[25] M. Galloway, "Texture analysis using gray level run lengths," Computer Graphics and Image Processing, vol. 4, pp. 172-179, 1975.

[26] S. Kim, K. Choi, and D. Lee, "Texture classification using run difference matrix," in Proc. of IEEE 1991 Ultrasonics Symposium, vol. 2, December 1991, pp. 1097-1100.

[27] H. Yoshida, D. Casalino, B. Keserci, A. Coskun, O. Ozturk, and A. Savranlar, "Wavelet-packet-based texture analysis for differentiation between benign and malignant liver tumours in ultrasound images," Physics in Medicine and Biology, vol. 48, pp. 3735-3753, 2003.

[28] N. Abbadeni, N. Ziou, and D. Wang, "Autocovariance-based perceptual textural features corresponding to human visual perception," in Proc. of 15th International Conference on Pattern Recognition, vol. 3, 2000, pp. 901-904.

[29] R. Yager, "On ordered weighted averaging aggregation operators in multicriteria decisionmaking," IEEE Trans. on SMC, vol. 18, no. 1, pp. 183-190, 1988.

[30] J. Russ, The Image Processing Handbook, 3rd ed. CRC Press and IEEE Press, 1999. 